\title{
Intrauterine growth and the maturation process of adrenal function
}

\author{
Sachiko Iwata ${ }^{1}$, Masahiro Kinoshita ${ }^{2}$, Hisayoshi Okamura ${ }^{2}$, Kennosuke Tsuda ${ }^{1}$, Mamoru Saikusa ${ }^{2}$, Eimei \\ Harada $^{2}$, Shinji Saitoh ${ }^{1}$, Osuke Iwata ${ }^{\text {Corresp. } 1}$ \\ ${ }^{1}$ Center for Human Development and Family Science, Department of Neonatology and Pediatrics, Nagoya City University Graduate School of Medical \\ Sciences, Nagoya, Aichi, Japan \\ ${ }^{2}$ Centre for Developmental and Cognitive Neuroscience, Department of Paediatrics and Child Health, Kurume University School of Medicine, Kurume, \\ Fukuoka, Japan
}

Corresponding Author: Osuke Iwata

Email address: o.iwata@med.nagoya-cu.ac.jp

Backgrounds. Environmental factors during early life alter the hypothalamus-pituitary-adrenal axis regulation and increase the risk of diseases in later life. However, adrenal function at each developmental stage has not fully been investigated in relation to pathological antenatal conditions. Cortisol levels of newborns with intrauterine growth restriction are elevated during the neonatal period, however, when studied during early childhood, cortisol levels are reduced compared with their peers, suggesting that the hypothalamus-pituitary-adrenal axis regulation might be altered from activation to suppression, the timing of which remains uncertain.

Aim. The aim of this study was to assess the presence of an interaction between intrauterine growth and postnatal age on cortisol levels in newborns hospitalised at a neonatal intensive care unit.

Methods. We performed a secondary analysis using a dataset from saliva samples of 62 newborns collected between 30 and 40 weeks corrected age. Interactions between postnatal age and clinical variables with regard to cortisol levels were assessed.

Results. The z-score of the birth weight and intrauterine growth restriction showed significant interactions with postnatal age on cortisol levels; cortisol levels were higher $\leq 5$ days of birth and lower $>14$ days of birth than those in their peers without intrauterine growth restriction.

Conclusion. The adrenal function of newborns with intrauterine growth restriction might be altered from activation to suppression within the first several weeks of life. Longitudinal studies need to address when/how intrauterine growth restriction alters adrenal functions, and how these responses are associated with diseases during adulthood. 
Title:

Intrauterine growth and the maturation process of adrenal function

\section{Authors:}

Sachiko Iwata, MD, PhD1, Masahiro Kinoshita, MD, PhD2, Hisayoshi Okamura, PhD2, Kennosuke Tsuda, MD, PhD1, Mamoru Saikusa, MD2, Eimei Harada, MD, PhD2, Shinji Saitoh, $\mathrm{MD}, \mathrm{PhD} 1$, Osuke Iwata, MD, PhD1,2

\section{Affuliations:}

10 1. Center for Human Development and Family Science, Department of Neonatology and

11 Pediatrics, Nagoya City University Graduate School of Medical Sciences, Nagoya, Aichi, Japan.

13 2. Centre for Developmental and Cognitive Neuroscience, Department of Paediatrics and Child 14 Health, Kurume University School of Medicine, Fukuoka, 830-0011 Japan.

\section{Correspondence to:}

\section{Dr Osuke Iwata}

18 Center for Human Development and Family Science, Department of Neonatology and Pediatrics, 19 Nagoya City University Graduate School of Medical Sciences, 1 Kawasumi, Mizuho-Cho, 20 Mizuho-ku, Nagoya, 467-8601 Japan.

21 E-mail: o.iwata@ucl.ac.ukＴel: +81 52 853-8246_ Fax: +81 52 842-3449 
Backgrounds. Environmental factors during early life alter the hypothalamus-pituitary-adrenal axis regulation and increase the risk of diseases in later life. However, adrenal function at each developmental stage has not fully been investigated in relation to pathological antenatal conditions. Cortisol levels of newborns with intrauterine growth restriction are elevated during the neonatal period, however, when studied during early childhood, cortisol levels are reduced compared with their peers, suggesting that the hypothalamus-pituitary-adrenal axis regulation might be altered from activation to suppression, the timing of which remains uncertain.

Aim. The aim of this study was to assess the presence of an interaction between intrauterine growth and postnatal age on cortisol levels in newborns hospitalised at a neonatal intensive care unit.

Methods. We performed a secondary analysis using a dataset from saliva samples of 62 newborns collected between 30 and 40 weeks corrected age. Interactions between postnatal age and clinical variables with regard to cortisol levels were assessed.

Results. The z-score of the birth weight and intrauterine growth restriction showed significant interactions with postnatal age on cortisol levels; cortisol levels were higher $\leq 5$ days of birth and lower $>14$ days of birth than those in their peers without intrauterine growth restriction. Conclusion. The adrenal function of newborns with intrauterine growth restriction might be altered from activation to suppression within the first several weeks of life. Longitudinal studies need to address when/how intrauterine growth restriction alters adrenal functions, and how these responses are associated with diseases during adulthood. 
Introduction

During pregnancy, plasma cortisol levels markedly change with gestational stage (Mastorakos \& Ilias 2003). However, the influence of maternal glucocorticoid on the foetus is limited because of the activation of placental 11- $\beta$-hydroxysteroid dehydrogenase 2 (11- $\beta$-HSD2) and subsequent conversion of cortisol to biologically inactive cortisone (Benediktsson et al. 1997). Clinical conditions such as maternal infection, strict diet, hypoxia, and foetal stress attenuate placental 11$\beta$-HSD2 activity (Hardy \& Yang 2002), which may increase foetal cortisol levels. Foetal exposure to excessive cortisol might induce pathological conditions such as intrauterine growth restriction (IUGR) and preterm labour (Kajantie et al. 2003; Stewart et al. 1995), which are associated with increased risks of short-term mortality and a range of long-term morbidities (Hack et al. 2002; Hutchinson et al. 2013; Murray et al. 2015; Pallotto \& Kilbride 2006). Increasing evidence suggests that homeostatic regulation is modified by environmental factors during early life, which is recognised as antenatal programming (Barker et al. 1990). Physiological stress response systems, particularly the hypothalamus-pituitary-adrenal (HPA) axis, have been suggested to play a central pathophysiological role in antenatal programming. Although stress-response actions may provide short-term benefits, the resultant alteration of the HPA axis may yield modified responses that result in the development of disease later in life.

Several studies have shown associations between perinatal physiological stress and attenuated adrenal function during childhood. For example, IUGR and preterm birth are associated with attenuated cortisol secretion at ages between 6 months and 7 years (Brummelte et al. 2015; FattalValevski et al. 2005; Ruys et al. 2017). In contrast, when studied during the neonatal period, paradoxically increased cortisol levels were observed in association with IUGR and preterm birth (Heckmann et al. 1999; Kajantie et al. 2003; Mericq et al. 2009; Stewart et al. 1995), suggesting that the HPA axis regulation might be altered from activation to suppression at some point during infancy or early childhood. However, thus far, few studies have addressed temporal changes in adrenal function following birth in association with exposure to pathological conditions in utero.

Using a cohort of newborns hospitalised in a neonatal intensive care unit (NICU), we have previously reported that lower cortisol levels were associated with clinical events suggestive of unfavourable foetal/postnatal conditions such as exposure to maternal pregnancy-induced hypertension (PIH), caesarean delivery, and preterm birth (Kinoshita et al. 2016). Using the same dataset with these studies, we performed a secondary analysis to assess whether maturational changes in cortisol levels after birth vary with the presence of IUGR and other clinical variables suggestive of foetal physiological stress.

Ethics approval and consent

\section{Material and Methods}

This study was conducted in compliance with the Declaration of Helsinki and with the approval of the Ethics Committee of Kurume University School of Medicine (13142). Written informed consent was obtained from one parent of each participating newborn.

This cross-sectional observational study was conducted as part of a project that investigated the independent variables of cortisol levels and their diurnal patterns (Kinoshita et al. 2016; Kinoshita et al. 2018). In an exploratory analysis of the dataset, the foetal-type diurnal pattern was observed on the basis of salivary cortisol levels, which were entrained in antiphase to the adult rhythm. Furthermore, the analysis found that lower cortisol levels were associated with older postnatal age, maternal PIH, caesarean delivery, preterm delivery, mechanical ventilation required at birth, and 
96 independence from ventilation support on the study day (Kinoshita et al. 2016). Feeding-induced

97 elevation of cortisol levels was also noted, which was more prominent following oral feeding and

98 was reduced with prolonged feeding (Kinoshita et al. 2018).

99

100

Study population

Detailed data on patient recruitment, sample collection, and cortisol assays have been reported previously (Kinoshita et al. 2016; Kinoshita et al. 2018). In brief, 65 newborns hospitalised in a tertiary NICU were recruited between 30 and 40 weeks (corrected age). Newborns, who were diagnosed with major congenital anomalies or chromosomal aberrations, who had undergone phototherapy within 24 hours previously, who had not been weaned from invasive mechanical ventilation and continuous intravenous infusion, and who had received glucocorticoid replacement therapy within 1 week previously, were excluded. Data collection was repeated up to two times for each patient, with a minimum interval of 7 days.

Sample collection and assay

Saliva was collected before and 1 hour after regular feeding at 10:00 and 19:00 using an absorbent swab (SalivaBio; Salimetrics LLC, State College, PA, USA). A cortisol assay was performed using an enzyme immunoassay kit (high-sensitivity salivary cortisol enzyme-linked immunosorbent assay kit; Salimetrics LLC). The limit of detection of this assay in our laboratory was $0.19 \mathrm{nmol} / \mathrm{L}$, and the intra- and inter-assay coefficients of variation were $5.43 \%$ and $6.41 \%$, respectively.

\section{Clinical variables}

Clinical information was obtained from electronic patient records, including maternal age, maternal body weight and body-mass index at the time of delivery, gestational age, delivery mode, intravenous tocolysis, premature rupture of membranes, histopathologically confirmed chorioamnionitis, PIH, multiple gestations, antenatal glucocorticoid administration, birth weight and its standard score calculated against the standard Japanese birth weight for gestational age (Itabashi et al. 2014), IUGR less than the $10^{\text {th }}$ percentile of the norm, sex, Apgar scores, requirement for respiratory support at birth, and predominant type of milk (formula or breast milk) on the day of saliva collection.

\section{Data analysis}

Values are presented as mean (standard deviation), unless otherwise specified. In the current study, only saliva samples collected before (but not after) feeding at 10:00 and 19:00 were considered because of the known influence of feeding on the cortisol levels (Kinoshita et al. 2018). Interactions between the clinical variables and postnatal age at saliva collection and their relation to salivary cortisol levels were assessed using the generalised estimating equations with gamma distribution modelling (SPSS ver. 20, IBM, Armonk, NY, USA). To minimise the potential influence of adrenal diurnal patterns and the bias from repeated sampling, the timing of saliva sampling (morning/evening and the study date when studied on two different days) was incorporated as a within-subject factor. Statistical significance was assumed when $\mathrm{p}<0.003$, after correcting for multiple comparisons of 18 variables. For combinations of categorical variables with significant interactions, the simple effect test was performed for cortisol levels among three postnatal ages of $\leq 5$ days, between 6 and 14 days, and $>14$ days (defined by the upper and lower quartile) for better clinical translation. 
142

143

144

145

146

147

148

149

150

151

152

153

154

155

156

157

158

159

160

161

162

163

164

165

166

167

168

169

170

171

172

173

174

175

176

177

178

179

180

181

182

183

184

185

186

187

\section{Results}

Out of the 65 newborns, two with an insufficient volume of saliva and one who was subsequently diagnosed with a major chromosomal aberration were excluded from the analysis. Subsequently, 124 samples from 62 cumulative newborns (corrected age, 31.7-39.5 weeks; postnatal age, 1-92 days) who were hospitalised because of low birth weight ( $\mathrm{n}=55)$, maternal hyperthyroidism or hypothyroidism $(n=2)$, or maternal gestational diabetes mellitus $(n=5)$ were considered (Table 1 and Dataset S1).

The main effects of potential antenatal and postnatal physiological stressors on the cortisol levels have been reported previously (See the second paragraph of the Material and Methods section for the brief summary of findings) (Kinoshita et al. 2016). Significant interactions were observed between postnatal age and the standard score of the body weight (standardised partial regression coefficient, 1.008; 95\% confidence interval, 1.004-1.012; $\mathrm{p}<0.001$ ), and postnatal age and IUGR (standardised partial regression coefficient, $0.981 ; 95 \%$ confidence interval, 0.968 $0.993 ; \mathrm{p}=0.002)($ Table 2$)$.

When the simple effect test was performed for newborns with/without IUGR, the cortisol levels were associated with postnatal age in newborns with (but not without) IUGR; cortisol levels at $>14$ days were lower than those at $\leq 5$ days and between 6 and 14 days (Fig. 1). Subsequently, IUGR newborns showed higher cortisol levels at $\leq 5$ days and paradoxically lower cortisol levels at $>14$ days than their peers (both $\mathrm{p}<0.001$ ).

\section{Discussion}

Our study suggested that foetal growth is associated with the relationship between postnatal age and cortisol levels in NICU newborns; cortisol levels of newborns with IUGR were higher soon after birth and lower after two weeks compared to those in their peers without IUGR. These findings build on our previous observations from the same dataset, that maternal PIH, caesarean delivery, and preterm birth are associated with lower cortisol levels in newborns hospitalised in the NICU (Kinoshita et al. 2016).

IUGR and adrenal function after birth

IUGR is associated with an increased incidence of both short-term and long-term morbidities (Murray et al. 2015, Pallotto 2006 Clin Obstet Gynecol PMID: 16721105). In newborns with IUGR, the expression of placental 11- $\beta$-HDS2 is decreased (Stewart et al. 1995), leading to increased cord blood cortisol levels in newborns with low body/placental weight (Heckmann et al. 1999; Mericq et al. 2009). Increased plasma cortisol levels and angiotensin II receptor subtype 1 expression induce constriction of peripheral vessels, leading to growth restriction of the foetal organs (Lanz et al. 2003). Studies further highlighted that antenatal exposure of the foetus to increased cortisol levels contributes to antenatal programming of the HPA axis and other systems, the influence of which is observed throughout (and even after) the developmental process (Barker et al. 1990; Hales et al. 1991).

Studies involving children and adults found associations between low birth weight and increased basal cortisol levels (Clark et al. 1996; Martinez-Aguayo et al. 2012). However, when assessed during early childhood, there were conflicting findings, showing no association (Dahlgren et al. 1998; Kajantie et al. 2002; Tenhola et al. 2002) or positive associations 
188 (Elhassan et al. 2015; Fattal-Valevski et al. 2005) between body size at birth and cortisol levels. 189 These inconsistent findings may be derived from the relatively wide age range when cortisol 190 levels were assessed, and the heterogeneous backgrounds of the population, for which the 191 influence of the birth weight was evaluated without incorporating gestational age. Our current 192 data suggest the potential presence of dynamic temporal changes in the adrenal function of IUGR which causes plasma cortisol levels to diminish rapidly after birth when maternal cortisol is no longer provided.

Because of the observations suggesting accelerated organ maturation of IUGR newborns (Amiel-Tison et al. 2004), the rapid postnatal decrease in cortisol levels in IUGR newborns might merely be a hint of the precocious maturation of the HPA axis that occurs with the earlierthan-usual physiological postnatal decrease in cortisol levels. This speculation might be relevant considering previous findings of attenuated HPA functioning during infancy and activated HPA functioning during adolescence and thereafter in IUGR newborns (Clark et al. 1996; FattalValevski et al. 2005; Martinez-Aguayo et al. 2012).

Limitations of the study

We did not serially obtain multiple saliva samples; temporal changes in cortisol levels were only extrapolated from cross-sectional findings. Because newborns were recruited when their feeding was established, preterm newborns had older postnatal ages than did their near-term/term peers at the time of the study. Our findings need to be re-assessed in future studies, which serially collect saliva samples from the first day of life to at least four weeks. IUGR newborns in the current study cohort exclusively had asymmetrical IUGR, presumably because symmetrical IUGR was excluded from the study cohort when newborns with major congenital anomalies were excluded.

\section{Conclusions}

Cortisol levels of newborns were associated with both postnatal age and intrauterine growth. Cortisol levels of newborns with IUGR were higher soon after birth and lower after two weeks than their peers without IUGR. It is possible to speculate that intrauterine growth may alter agespecific cortisol levels via deactivation of the HPA axis within first weeks of life. Future studies should address the impact of IUGR and other perinatal physiological stressors (and their level and duration) on the adrenal function and the development of diseases during adulthood. The potential benefits of care designed to minimise perinatal physiological stressors in these vulnerable newborns also need to be evaluated. 
224 The authors thank the patients who participated in the study and their parents for their cooperation, 225 the nurses of the Neonatal Intensive Care Unit, Kurume University Hospital for their support, and 226 Ms Chiho Yoshii and Chiaki Ueno for their consistent support.

227

228

229 
230

231

232

233

234

235

236

237

238

239

240

241

242

243

244

245

246

247

248

249

250

251

252

253

254

255

256

257

258

259

260

261

262

263

264

265

266

267

268

269

270

271

272

273

274

275

276

277

278

279

280

\section{References}

Amiel-Tison C, Cabrol D, Denver R, Jarreau PH, Papiernik E, and Piazza PV. 2004. Fetal adaptation to stress. Part I: acceleration of fetal maturation and earlier birth triggered by placental insufficiency in humans. Early Hum Dev 78:15-27. 10.1016/j.earlhumdev.2004.03.002

Barker DJ, Bull AR, Osmond C, and Simmonds SJ. 1990. Fetal and placental size and risk of hypertension in adult life. BMJ 301:259-262.

Benediktsson R, Calder AA, Edwards CR, and Seckl JR. 1997. Placental 11 beta-hydroxysteroid dehydrogenase: a key regulator of fetal glucocorticoid exposure. Clin Endocrinol (Oxf) 46:161-166.

Brummelte S, Chau CM, Cepeda IL, Degenhardt A, Weinberg J, Synnes AR, and Grunau RE. 2015. Cortisol levels in former preterm children at school age are predicted by neonatal procedural painrelated stress. Psychoneuroendocrinology 51:151-163. 10.1016/j.psyneuen.2014.09.018

Clark PM, Hindmarsh PC, Shiell AW, Law CM, Honour JW, and Barker DJ. 1996. Size at birth and adrenocortical function in childhood. Clin Endocrinol (Oxf) 45:721-726.

Dahlgren J, Boguszewski M, Rosberg S, and Albertsson-Wikland K. 1998. Adrenal steroid hormones in short children born small for gestational age. Clin Endocrinol (Oxf) 49:353-361.

Elhassan ME, Miller AL, Vazquez DM, and Lumeng JC. 2015. Associations of Prenatal and Perinatal Factors with Cortisol Diurnal Pattern and Reactivity to Stress at Preschool Age Among Children Living in Poverty. J Clin Res Pediatr Endocrinol 7:114-120. 10.4274/jcrpe.1685

Fattal-Valevski A, Toledano-Alhadef H, Golander A, Leitner Y, and Harel S. 2005. Endocrine profile of children with intrauterine growth retardation. J Pediatr Endocrinol Metab 18:671-676.

Hack M, Flannery DJ, Schluchter M, Cartar L, Borawski E, and Klein N. 2002. Outcomes in young adulthood for very-low-birth-weight infants. NEnglJMed 346:149-157.

Hales CN, Barker DJ, Clark PM, Cox LJ, Fall C, Osmond C, and Winter PD. 1991. Fetal and infant growth and impaired glucose tolerance at age 64. BMJ 303:1019-1022.

Hardy DB, and Yang K. 2002. The expression of 11 beta-hydroxysteroid dehydrogenase type 2 is induced during trophoblast differentiation: effects of hypoxia. J Clin Endocrinol Metab 87:3696-3701. 10.1210/jcem.87.8.8720

Heckmann M, Wudy SA, Haack D, and Pohlandt F. 1999. Reference range for serum cortisol in well preterm infants. Arch Dis Child Fetal Neonatal Ed 81:F171-174.

Hutchinson EA, De Luca CR, Doyle LW, Roberts G, and Anderson PJ. 2013. School-age outcomes of extremely preterm or extremely low birth weight children. Pediatrics 131:e1053-1061. 10.1542/peds.2012-2311

Itabashi K, Miura F, Uehara R, and Nakamura Y. 2014. New Japanese neonatal anthropometric charts for gestational age at birth. Pediatr Int 56:702-708. 10.1111/ped.12331

Kajantie E, Dunkel L, Turpeinen U, Stenman UH, Wood PJ, Nuutila M, and Andersson S. 2003. Placental 11 beta-hydroxysteroid dehydrogenase-2 and fetal cortisol/cortisone shuttle in small preterm infants. J Clin Endocrinol Metab 88:493-500. 10.1210/jc.2002-021378

Kajantie E, Phillips DI, Andersson S, Barker DJ, Dunkel L, Forsen T, Osmond C, Tuominen J, Wood PJ, and Eriksson J. 2002. Size at birth, gestational age and cortisol secretion in adult life: foetal programming of both hyper- and hypocortisolism? Clin Endocrinol (Oxf) 57:635-641.

Kinoshita M, Iwata S, Okamura H, Saikusa M, Hara N, Urata C, Araki Y, and Iwata O. 2016. Paradoxical diurnal cortisol changes in neonates suggesting preservation of foetal adrenal rhythms. Sci Rep 6:35553. 10.1038/srep35553

Kinoshita M, Iwata S, Okamura H, Tsuda K, Saikusa M, Harada E, Yamashita Y, Saitoh S, and Iwata O. 2018. Feeding-induced cortisol response in newborn infants. J Clin Endocrinol Metab. 10.1210/jc.2018-01052

Lanz B, Kadereit B, Ernst S, Shojaati K, Causevic M, Frey BM, Frey FJ, and Mohaupt MG. 2003. Angiotensin II regulates 11beta-hydroxysteroid dehydrogenase type 2 via AT2 receptors. Kidney Int 64:970-977. 10.1046/j.1523-1755.2003.00192.x

Martinez-Aguayo A, Aglony M, Bancalari R, Avalos C, Bolte L, Garcia H, Loureiro C, Carvajal C, 
281

282

283

284

285

286

287

288

289

290

291

292

293

294

295

296

297

298

299

300

301

302

303

304

305

306
Campino C, Inostroza A, and Fardella C. 2012. Birth weight is inversely associated with blood pressure and serum aldosterone and cortisol levels in children. Clin Endocrinol (Oxf) 76:713-718. $10.1111 / \mathrm{j} .1365-2265.2011 .04308 . \mathrm{x}$

Mastorakos G, and Ilias I. 2003. Maternal and fetal hypothalamic-pituitary-adrenal axes during pregnancy and postpartum. Ann N Y Acad Sci 997:136-149.

Mericq V, Medina P, Kakarieka E, Marquez L, Johnson MC, and Iniguez G. 2009. Differences in expression and activity of 11 beta-hydroxysteroid dehydrogenase type 1 and 2 in human placentas of term pregnancies according to birth weight and gender. Eur J Endocrinol 161:419-425. 10.1530/EJE09-0308

Murray E, Fernandes M, Fazel M, Kennedy SH, Villar J, and Stein A. 2015. Differential effect of intrauterine growth restriction on childhood neurodevelopment: a systematic review. BJOG 122:10621072. 10.1111/1471-0528.13435

Pallotto EK, and Kilbride HW. 2006. Perinatal outcome and later implications of intrauterine growth restriction. Clin Obstet Gynecol 49:257-269.

Ruys CA, van der Voorn B, Lafeber HN, van de Lagemaat M, Rotteveel J, and Finken MJJ. 2017. Birth weight and postnatal growth in preterm born children are associated with cortisol in early infancy, but not at age 8 years. Psychoneuroendocrinology 82:75-82. 10.1016/j.psyneuen.2017.05.007

Stewart PM, Rogerson FM, and Mason JI. 1995. Type 211 beta-hydroxysteroid dehydrogenase messenger ribonucleic acid and activity in human placenta and fetal membranes: its relationship to birth weight and putative role in fetal adrenal steroidogenesis. J Clin Endocrinol Metab 80:885-890. 10.1210/jcem.80.3.7883847

Tenhola S, Martikainen A, Rahiala E, Parviainen M, Halonen P, and Voutilainen R. 2002. Increased adrenocortical and adrenomedullary hormonal activity in 12-year-old children born small for gestational age. J Pediatr 141:477-482. 10.1067/mpd.2002.126923 


\section{Figure legend}

308

309 Figure 1: Box plot depicting the interaction between postnatal age and intrauterine growth 310 restriction on cortisol levels

IUGR newborns showed higher cortisol levels at $\leq 5$ days and lower cortisol levels at $>14$ days

313 compared with their peers. Association between postnatal age and cortisol levels was observed for 314 newborns with IUGR, but not for those without IUGR.

315 Statistical findings are from the simple effect test with Bonferroni correction.

316 Symbols: box, first and third quartiles; bold line, median; perpendicular line, range without 317 outliers; and open circle, extreme outlier less than 1.5 times the interquartile range from the first 318 quartile.

319

320

Abbreviation: IUGR, intrauterine growth restriction.

321

322

323

324

325

326 


\section{Table 1 (on next page)}

Clinical backgrounds of the study cohort

Values are shown as number (\%) or mean \pm standard deviation. 
1 Clinical backgrounds of the study cohort

2

\begin{tabular}{|c|c|}
\hline Variables during pregnancy & \\
\hline \multicolumn{2}{|l|}{ Maternal and antenatal variables } \\
\hline Intravenous tocolysis & $51(82.3)$ \\
\hline Pregnancy-induced hypertension & $10(16.1)$ \\
\hline Antenatal glucocorticoid & $29(46.8)$ \\
\hline Premature rupture of membranes & $22(35.5)$ \\
\hline Chorioamnionitis & $18(29.0)$ \\
\hline Maternal age (years) & $31.0 \pm 6.2$ \\
\hline Maternal height $(\mathrm{cm})$ & $158.2 \pm 5.1$ \\
\hline Maternal body weight $(\mathrm{kg})$ & $61.1 \pm 11.1$ \\
\hline Maternal body-mass index & $24.4 \pm 4.1$ \\
\hline \multicolumn{2}{|l|}{ Variables at birth } \\
\hline Vaginal delivery & $26(41.9)$ \\
\hline Multiple births & $15(24.2)$ \\
\hline Male & $27(43.5)$ \\
\hline Gestational age (weeks) & $32.3 \pm 3.7$ \\
\hline Birth weight $(\mathrm{g})$ & $1671 \pm 579$ \\
\hline Standard score of the birth weight & $-0.55 \pm 1.13$ \\
\hline Intrauterine growth restriction & $20(32.3)$ \\
\hline Apgar score (1 min) & $6.4 \pm 2.5$ \\
\hline Apgar score (5 min) & $8.1 \pm 1.5$ \\
\hline Need for mechanical ventilation & $3150.0)$ \\
\hline Postnatal glucocorticoid & $7(11.3)$ \\
\hline \multicolumn{2}{|l|}{ Variables on the day of saliva sampling } \\
\hline Postnatal age (days) & $24.3 \pm 22.6$ \\
\hline Corrected age (weeks) & $35.8 \pm 1.3$ \\
\hline Predominant breast milk feeding & $37(59.7)$ \\
\hline
\end{tabular}

3 Values are shown as number (\%) or mean \pm standard deviation. 


\section{Table 2 (on next page)}

Effect of antenatal stressors on cortisol levels according to postnatal age

The p-values are from the generalised estimating equation (see "Data analysis" in "Material and Methods" for detail), and are presented for the main effects of each independent variable and postnatal age and their interaction. Statistical significance was assumed when $p<0.003$, after correcting for multiple comparisons of 18 variables. Abbreviations: $\beta$, standardised partial regression coefficient; IUGR, intrauterine growth restriction; PIH, pregnancy-induced hypertension; PNA, postnatal age; PROM, premature rupture of membranes; SD, standard deviation. 
1 Effect of antenatal stressors on cortisol levels according to postnatal age

\begin{tabular}{|c|c|c|c|c|c|c|c|c|}
\hline \multirow{3}{*}{ Clinical conditions } & & \multirow{3}{*}{$\begin{array}{c}\text { Cortisol } \\
(\mathrm{nmol} / \mathrm{L}) \\
\text { Mean (SD) }\end{array}$} & \multicolumn{2}{|c|}{ Main effects } & \multicolumn{4}{|c|}{ Interaction with PNA } \\
\hline & & & \multirow{2}{*}{$\begin{array}{c}\text { Condition } \\
\qquad p\end{array}$} & \multirow{2}{*}{$\begin{array}{c}\text { PNA } \\
\text { p }\end{array}$} & \multirow{2}{*}{$\begin{array}{c}\beta \\
\text { Mean }\end{array}$} & \multicolumn{2}{|c|}{$95 \% \mathrm{CI}$} & \multirow[t]{2}{*}{$\mathrm{p}$} \\
\hline & & & & & & lower & Upper & \\
\hline \multirow[t]{2}{*}{ Intravenous tocolysis } & Yes & $8.79(8.76)$ & 0.144 & 0.738 & 0.987 & 0.968 & 1.006 & 0.183 \\
\hline & No & $8.03(5.04)$ & & & & & & \\
\hline \multirow[t]{2}{*}{ PROM } & Yes & $9.24(9.52)$ & 0.393 & 0.006 & 0.994 & 0.979 & 1.009 & 0.457 \\
\hline & No & $8.14(6.51)$ & & & & & & \\
\hline \multirow[t]{2}{*}{ PIH } & Yes & $6.10(4.21)$ & 0.259 & $<0.001$ & 0.994 & 0.982 & 1.005 & 0.269 \\
\hline & No & $9.00(8.12)$ & & & & & & \\
\hline Maternal Age & & & 0.442 & 0.598 & 1.000 & 0.999 & 1.001 & 0.962 \\
\hline Maternal body-mass index & & & 0.027 & 0.004 & 1.002 & 1.000 & 1.003 & 0.023 \\
\hline Maternal body weight & & & 0.051 & 0.014 & 1.000 & 1.000 & 1.001 & 0.074 \\
\hline \multirow[t]{2}{*}{ Chorioamnionitis } & Yes & $8.41(6.65)$ & 0.541 & 0.003 & 1.009 & 0.997 & 1.022 & 0.151 \\
\hline & No & $8.58(8.11)$ & & & & & & \\
\hline \multirow[t]{2}{*}{ Antenatal glucocorticoid } & Yes & $7.29(6.15)$ & 0.819 & 0.008 & 0.993 & 0.979 & 1.008 & 0.367 \\
\hline & No & $9.62(8,72)$ & & & & & & \\
\hline \multirow[t]{2}{*}{ Postnatal glucocorticoid } & Yes & $6.43(4.42)$ & 0.870 & 0.112 & 1.005 & 0.985 & 1.026 & 0.618 \\
\hline & No & $8.80(7.99)$ & & & & & & \\
\hline Standard score of the birth weight & & & 0.022 & 0.004 & 1.008 & 1.004 & 1.012 & $<0.001$ \\
\hline \multirow[t]{2}{*}{ IUGR } & Yes & $9.23(7.92)$ & 0.144 & $<0.001$ & 0.981 & 0.968 & 0.993 & 0.002 \\
\hline & No & $8.20(7.60)$ & & & & & & \\
\hline \multirow[t]{2}{*}{ Multiple pregnancy } & Yes & $8.84(8.74)$ & 0.756 & 0.182 & 1.004 & 0.982 & 1.026 & 0.744 \\
\hline & No & $8.29(6.83)$ & & & & & & \\
\hline \multirow[t]{2}{*}{ Vaginal delivery } & Yes & $10.37(9.52)$ & 0.641 & 0.013 & 1.009 & 0.997 & 1.020 & 0.140 \\
\hline & No & $7.20(5.76)$ & & & & & & \\
\hline \multirow[t]{2}{*}{ Male sex } & Yes & $8.29(6.83)$ & 0.286 & 0.005 & 0.989 & 0.976 & 1.003 & 0.121 \\
\hline & No & $8.84(8.74)$ & & & & & & \\
\hline Gestational Age & & & 0.346 & 0.784 & 1.000 & 0.997 & 1.002 & 0.748 \\
\hline \multirow[t]{2}{*}{ 5-min Apgar score $<7$} & Yes & $6.30(4.56)$ & 0.295 & 0.059 & 1.008 & 0.992 & 1.024 & 0.338 \\
\hline & No & $8.86(8.02)$ & & & & & & \\
\hline \multirow[t]{2}{*}{ Mechanical ventilation } & Yes & $6.76(5.07)$ & 0.632 & 0.896 & 0.989 & 0.954 & 1.026 & 0.571 \\
\hline & No & $10.30(9.34)$ & & & & & & \\
\hline Breast milk & Yes & $6.88(5.52)$ & 0.061 & 0.032 & 1.006 & 0.992 & 1.020 & 0.371 \\
\hline
\end{tabular}


2

3 The p-values are from the generalised estimating equation (see "Data analysis" in "Material and Methods" for 4 detail), and are presented for the main effects of each independent variable and postnatal age and their interaction. 5 Statistical significance was assumed when $\mathrm{p}<0.003$, after correcting for multiple comparisons of 18 variables.

6 Abbreviations: $\beta$, standardised partial regression coefficient; IUGR, intrauterine growth restriction; PIH, 7 pregnancy-induced hypertension; PNA, postnatal age; PROM, premature rupture of membranes; SD, standard 8 deviation. 


\section{Figure 1}

Box plot depicting the interaction between postnatal age and intrauterine growth restriction on cortisol levels

IUGR newborns showed higher cortisol levels at $\leq 5$ days and lower cortisol levels at $>14$ days compared with their peers. Association between postnatal age and cortisol levels was observed for newborns with IUGR, but not for those without IUGR. Statistical findings are from the simple effect test with Bonferroni correction. Symbols: box, first and third quartiles; bold line, median; perpendicular line, range without outliers; and open circle, extreme outlier less than 1.5 times the interquartile range from the first quartile. Abbreviation: IUGR, intrauterine growth restriction. 


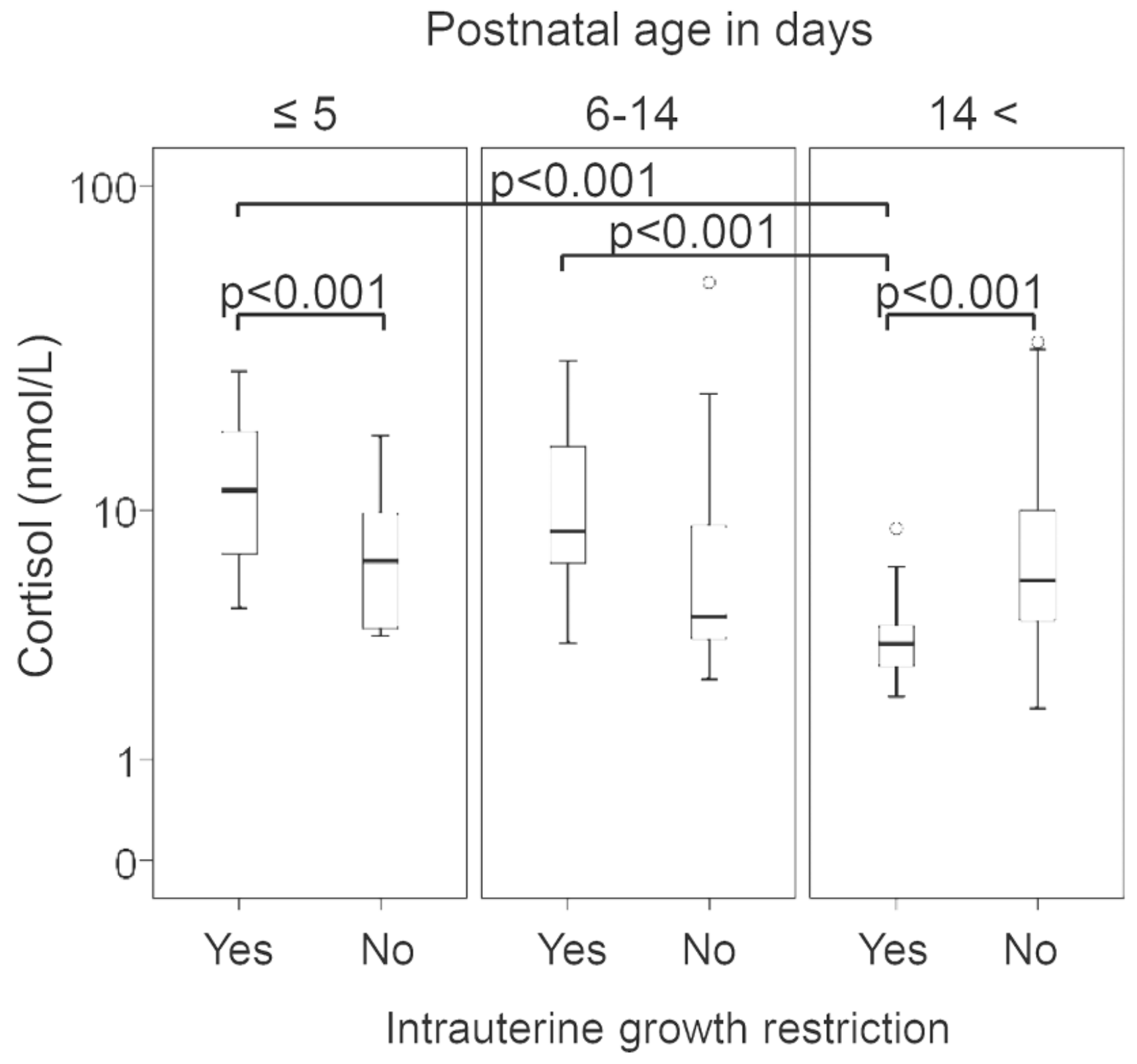

